\title{
The Prevalence of 23S rRNA Mutations in ML-Resistant $M$. pneumoniae Isolates to Clarithromycin in Patients with Respiratory Infections
}

\author{
Hanieh Big Mohammadi ${ }^{1}$, Iman Pouladi ${ }^{2}$, \\ Mohammad Reza Zolfaghari ${ }^{1}$, Mohammad Niakan*3
}

\begin{abstract}
Background: Mycoplasma pneumoniae is one of the widespread causes of community-acquired pneumonia (CAP). Over recent years, the widespread use of macrolides has led to the emergence of macrolide-resistant $M$. pneumoniae (MRMP) resulted from mutations at specific positions of domain $\mathrm{V}$ of the $23 S$ rRNA gene.

Methods: We collected 100 samples of throat swabs from patients with respiratory infections. After extraction of DNA from bacterial cell cultured in PPLO broth media using Roche kit (Germany), the PCR was performed on specific samples of $M$. pneumoniae using specific primers for $23 S r R N A$ gene. Afterwards, for positive samples, minimum inhibitory concentration (MIC) was determined using the broth microdilution with Clarithromycin. Finally, the PCR product was sequenced to detect mutations related to macrolide resistance in domain $\mathrm{V}$ of $23 S \mathrm{~S} R N A$.

Results: According to the analysis of the sequenced PCR product of $M$. pneumoniae $23 S \mathrm{rRNA}$ gene using Clustalw2 online software, one of the samples were shown to have a mutation at A2431G and G2491A positions. The MIC measurement also revealed that all isolates were sensitive to Clarithromycin, and there was no macrolide resistance to Clarithromycin in all isolates.

Conclusions: Sequence analysis of the $23 S$ rRNA gene in $M$. pneumoniae, revealed no macrolide resistance of $M$. pneumoniae to Clarithromycin. Thus, the use of these antibiotics should be restricted to prevent the development of macrolide-resistant $M$. pneumoniae in Iran.
\end{abstract}

Keywords: Clarithromycin, Macrolide resistance, Mycoplasma pneumonia, 23S rRNA gene.

\section{Introduction}

Mycoplasma pneumoniae (M. pneumoniae) is one of the most leading causes of communityacquired pneumonia (CAP) (1-3). M. pneumoniae Infections occur endemically worldwide, with the highest prevalence among children aged 5-15 years (4). In recent years, pneumonia caused by $M$. pneumoniae has shown an increasing trend (5). Previous studies have shown that $M$. pneumoniae accounts for up to 5\% of CAP cases (6). Although
M. pneumoniae is a self-limiting disease, some patients complain of progressive disease despite courses of antibiotic therapy (7-9). Macrolides (MLs) are used as the first-line treatment of $M$. pneumoniae infection (1-10). The duration of treatment of infections with ML-resistant $M$. pneumoniae (MRMP) is longer compared to treatment-sensitive infections (11-12). It was first reported in Japan, and then in other parts of the world, including the United States, Europe, East 
Asia, and the Middle East, respectively. The rate of MRMP has shown an annual increasing trend, especially in East Asian countries, including Japan, China, and Korea (12-13). In recent years, MRMP has become rampant in Asia, and has attracted the attention of scientists (1-3). The widespread use of macrolides has led to a rapid global emergence of MRMP as > $1 \%$ in Asia and $>2 \%$ in Europe (1). The resistance of $M$. pneumoniae to MLs has been shown to be caused by point mutations, nucleotide displacement, in domain $\mathrm{V}$ of the $23 S \mathrm{r} R N A$ gene (1-3). According to the results of previous studies over the past few years, $M$. pneumoniae strains with mutations in domain $\mathrm{V}$ of the $23 S \mathrm{rRNA}$ have been shown to have high levels of resistance to ML antibiotics in minimum inhibitory concentration (MIC) assay. Analysis of $\mathrm{V}$ domain sequence of $23 S r R N A$, revealed point mutations at some positions, such as A2063G, A2064G, and A2064C (14-15). As a result, studies have shown that $M$. pneumoniae have macrolide resistance and that all macrolideresistant $M$. pneumoniae strains isolated from patients with atypical pneumonia displayed point mutations in domain $\mathrm{V}$ of $23 S$ rRNA. The present study aimed to determine the prevalence of resistance of $M$. pneumoniae strains isolated from patients with respiratory infections to Clarithromycin due to $23 S$ rRNA mutation.

\section{Materials and methods \\ Subjects}

Totally, 100 samples of throat swab were collected from patients with atypical pneumonia admitted to Mostafa Khomeini and Khatamol-
Anbia hospitals of Tehran province in 2018. All patients were diagnosed with clinical symptoms of respiratory infections by a lung specialist, including weakness, lethargy, fatigue, persistent headache, dry cough, shortness of breath, diarrhea, sputum production, and muscle pain. The samples were taken from who did not have antibiotic therapy during the past month and filled up questioners and signed consent form.

\section{Sample collection}

Samples were taken from the upper throat of patients with respiratory infections using sterile cotton swabs, then were transferred to the laboratory in the transport PPLO broth medium. Then one $\mathrm{ml}$ of the transport medium was passed through the $0.45 \mu \mathrm{m}$ filter in the main Glucose PPLO broth medium in 5-10\% CO2, and then incubated at $35{ }^{\circ} \mathrm{C}$ for 3 weeks. In this study, $M$. pneumoniae (ATCC: 29342), was used as a control provided by the Molecular Biology Research Center of Baqiyatallah University of Medical Sciences.

\section{DNA extraction}

The Roche Co. kit (High Pure PCR Template Preparation Kit, Germany) was used to extract DNA from the samples were grown on the Glucose PPLO broth medium.

\section{Polymerase chain reaction (PCR)}

Following the extraction of genomic DNA, the Specific Primer-Polymerase Chain Reaction (SSP-PCR) was used to identify the genus Mycoplasma (23S rRNA gene) and $M$. pneumoniae strain ( $P 1$ gene) (Table 1$)$.

Table 1. Sequences of primers used in this study.

\begin{tabular}{|c|c|c|}
\hline Gene & Sequence & Product Size (Reference) \\
\hline$P 1$ & $\begin{array}{l}\text { FP: 5'- AAAGGAAGCTGACTCCGACA } \\
\text { RP: 5'-TGGCCTTGCGCTACTAAGTT }\end{array}$ & 450 bp (16) \\
\hline $23 S r R N A$ & $\begin{array}{l}\text { FP: 5‘- TAACTATAACGGTCCTAAGG } \\
\text { RP: 5‘- CGCTACAACTGGAGCATAAGA }\end{array}$ & 793 bp (17) \\
\hline
\end{tabular}


After Primer-BLAST on designated primers, the PCR was conducted at a final volume of $25 \mu$, including an initial denaturation at $94{ }^{\circ} \mathrm{C}$ for 5 min, 35 cycles comprised of denaturation at $94^{\circ} \mathrm{C}$ for 35 seconds, binding primer to the target DNA at $56{ }^{\circ} \mathrm{C}$ for 40 seconds, elongation at $72{ }^{\circ} \mathrm{C}$ for 45 seconds, as well as a final elongation at $72^{\circ} \mathrm{C}$ for 5 minutes, according to the protocol, for $M$. pneumoniae; and also another PCR at a final volume of $25 \mu \mathrm{l}$, constituted of an initial denaturation at $94{ }^{\circ} \mathrm{C}$ for $4 \mathrm{~min}, 33$ cycles comprised of denaturation at $94{ }^{\circ} \mathrm{C}$ for 45 seconds, binding primer to the target DNA at 55 ${ }^{\circ} \mathrm{C}$ for 45 seconds, elongation at $72{ }^{\circ} \mathrm{C}$ for 50 seconds, as well as a final elongation at $72{ }^{\circ} \mathrm{C}$ for 5 minutes, according to the protocol for the $23 \mathrm{~S}$ $r R N A$. The kit was obtained from Roche Co. (Germany) was used to extract the 23S rRNA. The PCR products (ATCC: 29342) were at last electrophoresed. Eventually, PCR products that were positive for $23 S \mathrm{rRNA}$ were sequenced with next-generation sequencing (NGS) to detect point mutation of domain $\mathrm{V}$ in $23 S$ rRNA, and the sequences of the $23 S r R N A$ gene were analyzed.

\section{Minimum inhibitory concentration (MIC) determination using microdilution}

The MIC was carried out by micro-dilution of Glucose PPLO broth, enriched with horse serum, and D-glucose in 96 wells of microplates on M. pneumoniae positive samples, following

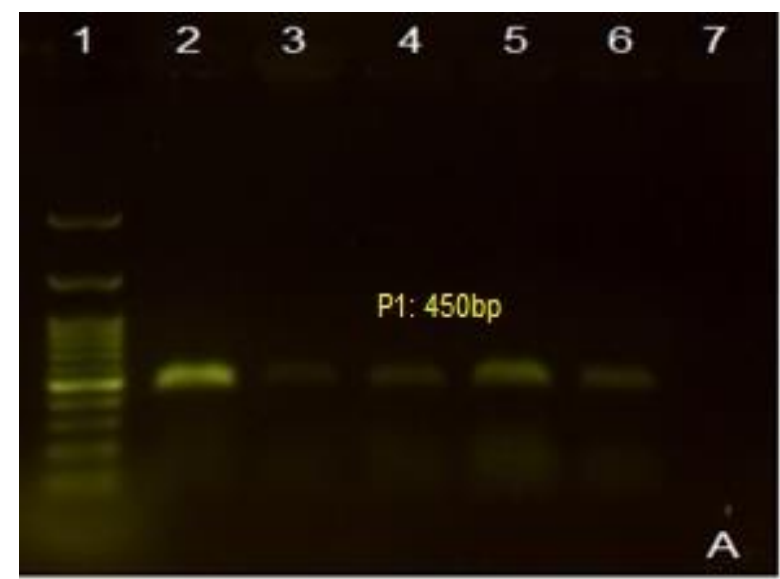

preparation of macrolide suspension of Clarithromycin. Then, the microplates containing colonies of $M$. pneumoniae were incubated at $37{ }^{\circ} \mathrm{C}$ for 5-6 days to determine the MIC of Clarithromycin. The MIC of Clarithromycin was measured and recorded for all M. pneumoniae positive samples. As such, the MIC of Clarithromycin was determined based on color changes of Glucose PPLO broth enriched with horse serum and Dglucose.

\section{Statistical analysis}

Data analysis was performed by using SPSS, version 20.0.

\section{Results}

\section{Demographic}

Of 100 assigned patients with respiratory infections, 48 (48\%) were males and 52 (52\%) were females. The average age of the participants was 53.62 years, ranging from 17 years to 85 years.

\section{Molecular detection of P1 and 23S rRNA}

The SSP-PCR showed $450 \mathrm{bp}$ amplification of $P 1$ in the standard strain and six samples $(6 \%)$ using the P1-specific primer of $M$. pneumoniae (Fig. 1A). Using $23 S$ rRNA-specific primers revealed a$793 \mathrm{bp}$ - amplicon in the standard strain and six samples (Fig. 1B).

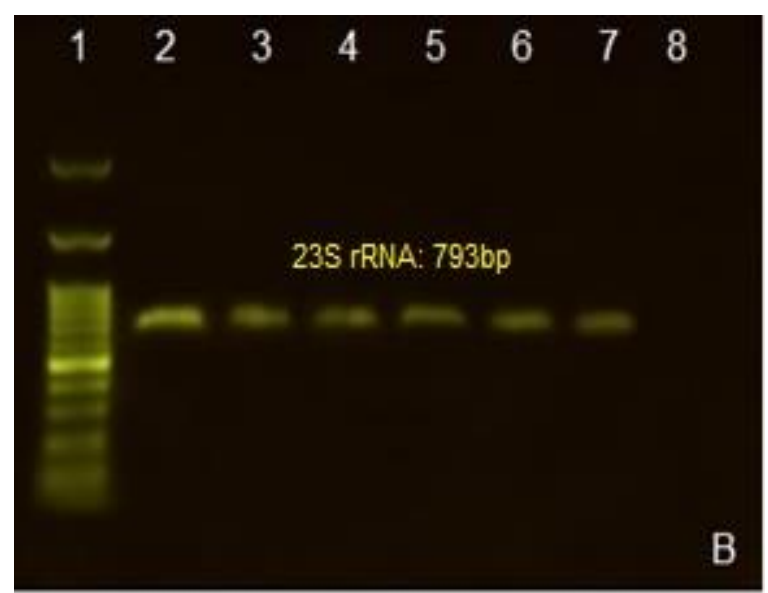

Fig. 1. A) PCR amplification of the $P 1$ gene. Lane 1: Ladder (100 bp), lane 7: negative control, lane 2: positive control ( $450 \mathrm{bp})$; lane 3-6: positive samples. B) PCR amplification of the $23 S$ rRNA gene. Lane 1: Ladder (100 bp), lane 8: negative control, lane 2: positive control (793 bp); lane 3-7: positive samples. 
Sequencing and Determination of minimum inhibitory concentration (MIC) in M. pneumoniae isolates

With analysis of the $23 S r R N A$ gene sequence, only one sample was found with a point mutation at A2431G and G2491A positions
(Fig. 2). Also, the measurement of MIC in all M. pneumoniae-positive samples using the broth micro-dilution method, indicated that all specimens were sensitive to Clarithromycin, and no ML resistance was reported (Table 2).

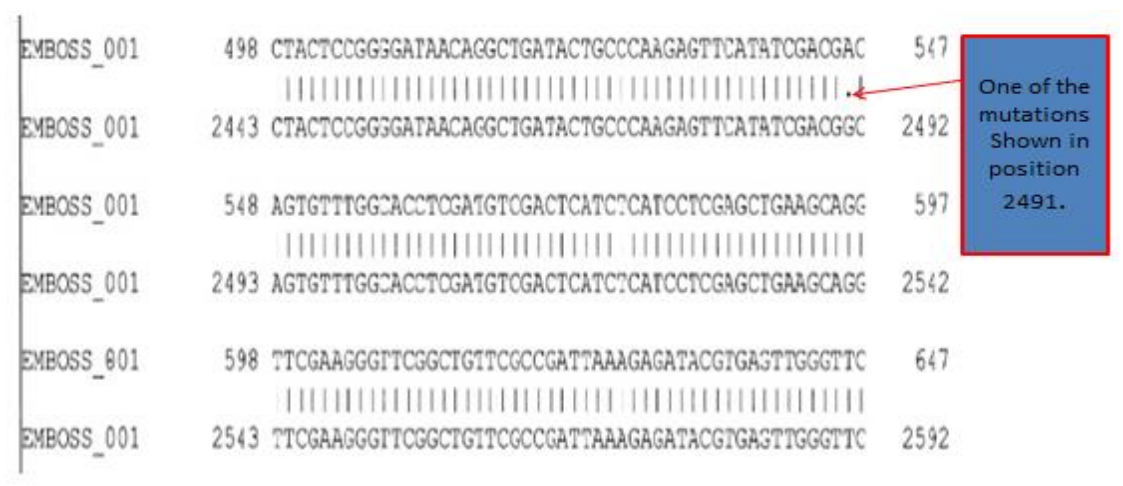

Fig. 2. A schematic representation of a mutation in $23 S r R N A$ genes in online clustalW2 software. One of the mutations is shown at position 2491.

Table 2. Determination of MIC of Clarithromycin in Mycoplasma pneumoniae- positive samples.

\begin{tabular}{lll}
\hline Clinical isolate & \multicolumn{1}{c}{ MIC } & Mutation \\
\hline A & 0.00025 & NO \\
B & 0.000125 & Yes \\
C & 0.001 & NO \\
D & 0.00025 & NO \\
E & 0.00025 & NO \\
F & 0.00025 & NO \\
Reference strain & 0.0039 & NO \\
\hline
\end{tabular}

\section{Discussion}

Mycoplasma Pneumoniae is a major cause of community-acquired pneumonia (5). Macrolide antibiotics are recommended as the first-line treatment for $M$. pneumoniae $(1,10)$. Extensive use of these antibiotics resulted in the emergence of ML-resistant isolates all over the world. Sharifi et al. conducted a study in Tabriz city on 200 patients and reported the prevalence of $M$. pneumoniae as $6 \%$, which was in accordance with our study. However, the prevalence of $\mathrm{M}$. pneumoniae reported in this study was significantly higher than the rate of $M$. pneumoniae reported in Ahvaz city and (3\%), Rasht city (1\%) (19). Lucier et al. found a number of ML-resistance $M$. pneumoniae at different positions in the $\mathrm{V}$ domain of the $23 \mathrm{~S} r R N A$ (2063 and 2064) with the displacement of A-G $(14,18)$. The prevalence of MRMP displays various patterns in different countries, which is attributed to the excessive use of MLs and the age of the patients (20).

Macrolides resistance is linked with a point mutation in domain $\mathrm{V}$ of the $23 \mathrm{~S} r R N A$ gene (nucleotide displacement at specific positions of 
domain $\mathrm{V}$ of $23 S \mathrm{rRNA}$ ). Mutation of $23 S \mathrm{rRNA}$ at $\mathrm{A} 2063 \mathrm{G}$ is the commonest mutation, followed by A2064G, which is mostly responsible for high ML resistance in $M$. pneumoniae $(18,21,22)$.

Here, the analysis of the sequence of $23 \mathrm{~S}$ rRNA demonstrated point mutations at $\mathrm{A} 2431 \mathrm{G}$ and G2491A only in one specimen. In addition, the broth micro-dilution MIC method indicated that all $M$. pneumonia- positive samples, were sensitive to Clarithromycin, and no ML resistance was reported. The frequency of MRMP amongst the participants was $0.6 \%$. Accordingly, the prevalence of MRMP was shown as 2\% (1/50) in Switzerland, 87.1\% (176/202) in Japan, 97\% (32/33) in China, 1.2\% (2/167) in Germany, and 9.8\% (5/51) in France, which were inconsistent with the results of our study (2). Hong and colleagues reported two cases of MRMP, and proposed that MRMP-positive cases had point mutation at $\mathrm{A} 2063 \mathrm{G}$ of domain $\mathrm{V}$ of $23 \mathrm{~S} r R N A$ (18). A study conducted in England (2015), reported mutations associated with ML resistance in $M$. pneumoniae- positive samples between September 2014 and September 2015. They stated that 43 of the total $60 \mathrm{M}$. pneumoniaepositive samples presented mutations in domain $\mathrm{V}$ of $23 S$ rRNA, with ML resistance in four samples $(9.3 \%)$. Those with point mutations in domain $\mathrm{V}$ of $23 S \mathrm{SRNA}$ were identified as MLresistant isolates (23), which was not in accordance with the results of our study. Haruki and colleagues recruited hospitalized children in Japan (2014) and detected 33 cases of $M$. pneumoniae by PCR, of whom 31 cases displayed resistance to MLs, and all cases were shown to have point mutation at 2063 and 2064 in domain $\mathrm{V}$ of $23 S$ rRNA (24). The contradiction in these

\section{References}

1. Eshaghi A, Memari N, Tang P, Olsha R, Farrell DJ, Low DE, et al. Macrolideresistant Mycoplasma pneumoniae in humans, Ontario, Canada, 2010-2011. Emerg Infect Dis. 2013;19(9).

2. Meyer Sauteura P, Bleischa B, Voitb A,4. Maurerb F, Rellya C, Berger C, et al. Survey of macrolide-resistant Mycoplasma pneumoniae in children with community-acquired pneumonia in Switzerland. Swiss Med Wkly. 2014;144:w14041. results may be due to the older age of the patients in our study. Liu and colleagues (2014) evaluated 580 samples of throat swab suspected for $M$. pneumoniae, and reported that 70 cases were ML-resistant. They also revealed mutation at 2063 and 2064 in domain $\mathrm{V}$ of $23 \mathrm{~S} r R N A$ in all specimens positive for $M$. pneumoniae (17). Zhou et al. (2015) also assessed 650 samples of throat swab and used the PCR. All sequences of the domain $\mathrm{V}$ of the $23 \mathrm{~S}$ rRNA gene were investigated. They proposed that 100 percent (71/71) of $M$. pneumoniae isolated from patients with community-acquired pneumonia were resistant to erythromycin, Clarithromycin, and azithromycin. Furthermore, all MLresistant strains of Mycoplasma pneumoniae displayed point mutation at $\mathrm{A} 2063 \mathrm{G}$ in the domain $\mathrm{V}$ of $23 S$ rRNA (25). These contradictory results can be attributed to the larger sample size of this study.

According to the analysis of the sequence of the $23 S r R N A$ gene, all positive samples for $M$. pneumoniae were not resistant to MLs. Besides, all point mutations in domain $\mathrm{V}$ of $23 S \mathrm{r} R N A$ at A2431G and G2491A were not linked with Clarithromycin resistance. In conclusion, in order to control or prevent the emergence of ML resistance among $M$. pneumoniae strains, the application of these antibiotics should be limited in Iran.

\section{Acknowledgment}

This paper was part of a master's thesis (grand no: 10430507942052). The authors would like to appreciate all staff members of the Microbiology department of Shahed University of Medical sciences.

3. Zhao F, Liu G, Wu J, Cao B, Tao X, He L, et al. Surveillance of Macrolide-Resistant Mycoplasma pneumoniae in Beijing, China, from 2008 to 2012. Antimicrob Agents Chemother.2013;57(3):1521-1523.

Babaei Z, Pouladi I, Ashtari A, Azimi G, Niakan M. The Prevalence of Atypical Pneumonia Caused by Mycoplasma pneumoniae (P1 gene) in Patients with Respiratory Infections by Culture and Molecular PCR Methods in Tehran, Iran. 
Jundishapur Journal of Microbiology. 2019; 12(11):e84174.

5. Song Q, Xu B, Shen K. Effects of bacterial and viral co-infections of mycoplasma pneumoniae pneumonia in children: analysis report from Beijing Children's Hospital between 2010 and 2014. Int J Clin Exp Med. 2015;8(9):15666-74.

6. Zhang Y, Mei S, Zhou Y, Yang D, Pan T, Chen $Z$, et al. TIPE2 negatively regulates mycoplasma pneumonia-triggered immune response via MAPK signaling pathway. 1ScIentIfIc RepoRts.2017;7(1):13319.

7. Zhou Y, Zhang Y, Sheng Y, Zhang L, Shen $Z$, Chen Z. More complications occur in macrolide-resistant than in macrolide-sensitive Mycoplasma pneumoniae pneumonia. Antimicrob Agents Chemother. 2014;58(2):1034-8.

8. Youn YS, Lee SC, Rhim JW, Shin MS, Kang $\mathrm{JH}$, Lee KY. Early Additional ImmuneModulators for Mycoplasma pneumoniae Pneumonia in Children: An Observation Study. Infect Chemother. 2014;46(4):239-47.

9. Meyer Sauteur P, Unger W, Nadal D, Berger C, Vink C, Rossum A. Infection with and Carriage of Mycoplasma pneumoniae in Children. Front Microbiol.2016;7:329.

10. Kawai Y, Miyashita N, Kubo M, Akaike H, Kato A, Nishizawa $\mathrm{Y}$, et al. Nationwide Surveillance of Macrolide-Resistant Mycoplasma pneumoniae Infection in Pediatric Patients. Antimicrob Agents Chemother.2013;57(8):4046-4049.

11. Saraya T. Mycoplasma pneumoniae infection: Basics. J Gen Fam Med. 2017;18(3):118-125.

12.Izumikawa $\mathrm{K}$, Izumikawa $\mathrm{K}$, Takazono $\mathrm{T}$, Kosai K, Morinaga Y, Nakamura S, et al. Clinical features, risk factors and treatment of fulminant Mycoplasma pneumoniae pneumonia: a review of the Japanese literature. J Infect Chemother. 2014;20:181-5.

13. Valentine-King MA, Cisneros K, James MO, Huigens RW 3rd, Brown MB. Turning the Tide against Antibiotic Resistance by Evaluating Novel, Halogenated Phenazine, Quinoline, and NH125 Compounds against Ureaplasma Species Clinical Isolates and Mycoplasma Type Strains. Antimicrob Agents Chemother. 2019;63(3):e02265-18.
14. Lucier TS, Heitzman K, Liu SK, Hu PC, et al. Transition mutations in the 23S rRNA of erythromycin-resistant isolates of Mycoplasma pneumoniae. Antimicrob Agents Chemother. 1995;39(12): 2770-3.

15. Liu Y, Ye X, Zhang H, Xu X, Li W, Zhu $\mathrm{D}$, et al. Antimicrobial Susceptibility of Mycoplasma pneumoniae Isolates and Molecular Analysis of Macrolide-Resistant Strains from Shanghai, China. Antimicrob Agents Chemother. 2009;53(5):2160-2.

16. Golmohammadi R, Ataee R A, Alishiri G H, Mirnejad R, Najafi A, Tate M, et al. Molecular Diagnosis of Mycoplasma pneumoniae in Synovial Fluid of Rheumatoid Arthritis Patients. Iran J Med Microbiol. 2014;8(1):1-8.

17. Liu X, Jiang Y, Chen X, Li J, Shi D, Xin D. Drug Resistance Mechanisms of Mycoplasma pneumoniae to Macrolide Antibiotics. Biomed Res Int. 2014;2014:1-7.

18. Hong JH, Chun JK, Uh Y, Oh KJ, Kim J, Yoon KJ. Two Cases of Mycoplasma pneumoniae Pneumonia with A2063G Mutation in the 23S rRNA Gene in Siblings. Ann Lab Med 2013; 33(1):65-8.

19. Sharifi S, Ghotaslo R, Aki MT, Soroush MH, Ansarian Kh, Shabanpour J, et al. Identification of respiratory infections caused by Mycoplasma pneumoniae with three methods of cultivation, ELISA and PCR. Medical Journal of Tabriz University. 2011; 33(3): 36-41.

20. Morozumi M, Iwata S, Hasegawa K, Chiba N, Takayanagi R, Matsubara K, et al. Increased macrolide resistance of Mycoplasma pneumoniae in pediatric patients with community-acquired pneumonia. Antimicrob Agents Chemother 2008;52(1):348-50.

21. Lin C, Li S, Sun H, Zhao H, Feng Y, Cao L, et al. Nested PCR-linked capillary electrophoresis and single-strand conformation polymorphisms for detection of macrolide-resistant Mycoplasma pneumoniae in Beijing, China. J Clin Microbiol. 2010;48(12):4567-72.

22. Morozumi M, Takahashi T, Ubukata K. Macrolide-resistant Mycoplasma pneumoniae: characteristics of isolates and clinical aspects of community-acquired pneumonia. J Infect Chemother. 2010;16(2):78-86. 
23. Brown RJ, Macfarlane-Smith L, Phillips $\mathrm{S}$, Chalker VJ, et al. Detection of macrolide resistant Mycoplasma pneumoniae in England, September 2014 to September 2015. Euro Surveill. 2015;20(48):30078.

24. Haruki K, Tomoyuki T, Ayano I, Tsuyoshi S, Tomoo F. Characteristics of hospitalized children infected with macrolide-resistant Mycoplasma pneumonia brazj infectdis.2014;18(3):294-299.

25. Zhou Z, Li X, Chen X, Luo F, Pan C, Zheng $\mathrm{X}$, et al. Macrolide-Resistant Mycoplasma pneumoniae in Adults in Zhejiang, China Antimicrob Agents Chemother. 